\title{
Biomarkers for Non-invasive Diagnosis of Non-alcoholic Steatohepatitis
}

\author{
Ancha Baranova • Zobair M. Younossi
}

Published online: 14 June 2011

(C) Springer Science+Business Media, LLC 2011

Reply:

We thank Dr. Yilmaz and co-authors for their interest in our research and their thoughtful comments describing common, but unavoidable, pitfalls on the road to developing clinically acceptable biomarkers for the diagnosis of non-alcoholic steatohepatitis (NASH) and related fibrosis. We are fully aware of these drawbacks, especially those related to the performance of a biomarker panel in comparison to tests containing only a single biomarker. It is important to note that some of the variables included in a panel of biomarker such as ours (presence of diabetes, gender, BMI, triglycerides, and AST) are routinely available in clinical practice [1]. Therefore, adding these parameters to a "biomarker panel" should not increase the associated costs. On the other hand, ELISA assays for the other components of the biomarker panel such as M30, M65, and TIMP1 should be readily available at a cost of around US \$35-40 (including the associated labor). We envision that biomarker panels such as the one reported by us should be fully validated and then be used as screening tools to identify patients at the highest likelihood of having NASH or NASH-related fibrosis.

In addition, we would like to add that there are other pitfalls that could plague development and validation of these noninvasive biomarkers for liver diseases. One of these traps is the necessity to rely on the liver biopsy as the "gold standard" for

A. Baranova $\cdot$ Z. M. Younossi $(\bowtie)$

Center for Liver Diseases,

Department of Medicine, Inova Fairfax Hospital,

3300 Gallows Road,

Falls Church, VA 22042, USA

e-mail: zobair.younossi@inova.org

\section{A. Baranova $\cdot$ Z. M. Younossi}

Betty and Guy Beatty Center for Integrated Research, Inova Health System,

Falls Church, VA, USA validation of newly developed biomarkers. It is important to bear in mind that the biopsy itself has an area under the curve (AUC), which could be derived from the "true gold standard" using an entire section of the liver [2]. As clearly demonstrated by Bedossa et al., liver biopsy sampling variability is especially prominent in the cases of discriminating stages of fibrosis [1]. In fact, the METAVIR scoring system which was used for staging fibrosis on a typical 15-mm liver biopsy core was correct approximately $65 \%$ of the times, reflecting an AUC of only 0.82 [1]. Because of this relatively low performance, using core liver biopsy as a "gold standard" for validating non-invasive biomarkers is difficult at best. The study by Bedossa et al. illustrates the difficulty of using core liver biopsies for the validation of non-invasive biomarkers. To overcome this problem, the future biomarker validation studies need to be designed in a way that allows assessment of multiple biomarkers performances against each other as well as against the "imperfect gold standard" of liver biopsy. Most importantly, these studies must be designed to assess long-term outcomes of patients with liver diseases. After all, what looks bad to the pathologist under the microscope or to the clinician reviewing patients' medical records must be bad for the patient. Despite the difficulty in their design, only validation studies assessing the long-term outcomes can be designed to answer these clinically important questions.

Conflict of Interest There are no conflicts of interest for any of the authors.

\section{References}

1. Younossi ZM, Page S, Rafiq N, et al. A biomarker panel for nonalcoholic steatohepatitis (NASH) and NASH-related fibrosis. Obes Surg. 2010;21:431-9.

2. Bedossa P, Dargère D, Paradis V. Sampling variability of liver fibrosis in chronic hepatitis C. Hepatology. 2003;38(6):1449-57. 